\title{
A Linear-Nonlinear Formulation of Einstein Equations for the Two-Body Problem in General Relativity
}

\author{
Pablo Laguna \\ Department of Astronomy \& Astrophysics and \\ Center for Gravitational Physics $\&$ Geometry \\ Penn State University, University Park, PA 16802, USA
}

(May 22, 2021)

\begin{abstract}
A formulation of Einstein equations is presented that could yield advantages in the study of collisions of binary compact objects during regimes between linear-nonlinear transitions. The key idea behind this formulation is a separation of the dynamical variables into i) a fixed conformal 3-geometry, ii) a conformal factor possessing nonlinear dynamics and iii) transverse-traceless perturbations of the conformal 3-geometry.
\end{abstract}

04.30.Nk

In the two-body problem in General Relativity, involving black holes and/or neutron stars, one can identify three main phases according to the extent to which nonlinear effects play a fundamental role. When the two bodies are sufficiently far apart, the binary system is in an Inspiral phase. During this phase, a Post-Newtonian approach to gravity provides a suitable framework. Loses due to gravitational radiation drive the binary system to the next stage, the Merging phase. During the merging, which includes the last few orbits and coalescence of the binary system, nonlinear effects become essential and eventually dominate the dynamics of the system; thus, the full set of Einstein fields equations is required. The last stage of the problem is the Ringdown phase. Here once again, the two-body problem can be treated perturbatively. In this situation, however, the system can be investigated as perturbations about the outcome of the collision, namely a single black hole or neutron star. This point of view is also known as the "close-limit" and has been shown to be a remarkable good approximation [1].

From the computational point of view, it is safe to say that the machinery, necessary to study the Inspiral and Ringdown phases of the problem, is available and reasonably well understood. On the other hand, in spite of advances in computer technology and numerical algorithms, the Merging phase of black hole and neutron star collisions remains essentially unsolved; that is, simulations that bridge the "gap" between the Inspiral and Ringdown stages are not yet available. The expectation is that this situation will prevail for the next few years. Furthermore, even if full nonlinear numerical simulations of the collision of compact objects are achieved [2], they will most likely not be able to cover, at least initially, the dynamical time-scales necessary to grab initial data from the Inspiral phase, evolve it through the Merging phase and provide initial data for Ringdown calculations. To get a sense of the formidable task involved, one only needs to keep in mind that, for numerical approaches using asymptotically inertial coordinates, the dynamical time-scale is $\sim M$. This time-scale should be compared with $\sim 1200 M$, the time-scale of numerical evolutions to cover the Inspiral-Ringdown gap. Because of this foreseen limitation, it is important to develop methodologies that directly address the intermediate regimes between the main phases discussed above.

The Inspiral-Merging intermediate regime has recently received significant attention. In the context of black hole collisions, this is called the "intermediate binary black holes" problem by Thorne and collaborators [3]. They have proposed an approach in which numerical calculations are performed in co-rotating coordinates with the binary system. In this co-rotating frame, the metric evolves on long time-scales, namely those of the inspiral scales. Furthermore, gauge conditions are chosen such that gravitational degrees of freedom that are not excited by radiation remain frozen.

Finding a reduced formulation of Einstein equations for the Inspiral-Merging as well as Merging-Ringdown intermediate regimes is the focus of this paper, namely a formulation tailored to the transitions between full non-linear and completely linear phases. Our working premise will be that, during those linear-nonlinear (LNL) transitions, "democracy" does not apply when determining the framework needed to handle the gravitational fields. That is, we expect that nonlinear effects are not equally turned on and off for all the field variables. There is evidence for such behavior. In the numerical study of neutron star binaries, Wilson and collaborators [4.5] used a simplified version of the Einstein equations in which only a reduced set of metric variables have nonlinear dynamics, with the remaining fields fixed in time. Although, a debate arose on one of the predictions from this work, namely the collapse of the neutron stars before their coalescence (see [6] for a resolution to this debate), this approach provides a reasonable approximation in the regime when the dynamics is dominated by volume (conformal) deformations and the energy radiated per orbit is much smaller than the energy of orbital motion. Our LNL formulation of Einstein equations builds upon Wilson's idea, allowing for more general background space-times and the presence of linear perturbations. The LNL is constructed by identifying, among the dynamical variables, those quantities that can be kept fixed, those that can be treated perturbatively and finally those that follow nonlinear dynamics. The hope is to arrive to a reduced 
system of Einstein equations that is not only more amenable to be handled numerically but also allows one to gain a better insight of the problem.

As customary in Numerical Relativity, the starting point is the $3+1$ or ADM form of Einstein equations [7]. Under the $3+1$ scheme, the space-time is viewed as the time history of a foliation of space-like hypersurfaces with intrinsic metric $\hat{h}_{a b}$, with Latin and Greek letters denoting spatial and space-time indices, respectively. Since most of our expressions will eventually involve conformal quantities, we will reverse the usual convention and denote physical quantities with hats and conformal quantities without hats. The $3+1$ line element takes the form:

$$
d s^{2}=-\hat{\alpha}^{2} d t^{2}+\hat{h}_{a b}\left(d x^{a}+\hat{\beta}^{a} d t\right)\left(d x^{b}+\hat{\beta}^{b} d t\right)
$$

with $\hat{\alpha}$ the lapse function characterizing proper time along the normals to the hypersurface, and $\hat{\beta}^{a}$ the shift vector, representing the freedom of relabeling spatial coordinate points in that surface. With the space-time metric given by (11), Einstein equations take the form:

$$
\begin{aligned}
\hat{R}+\hat{K}^{2}-\hat{K}_{a b} \hat{K}^{a b} & =0 \\
\hat{\nabla}_{b} \hat{K}^{a b}-\hat{\nabla}^{a} \hat{K} & =0 \\
\hat{\partial}_{o} \hat{h}_{a b} & =-2 \hat{\alpha} \hat{K}_{a b} \\
\hat{\partial}_{o} \hat{K}_{a b}+2 \hat{\alpha} \hat{K}_{a c} \hat{K}_{b}^{c}-\hat{\alpha} \hat{K}_{a b} \hat{K} & =\hat{\alpha}\left(\hat{R}_{a b}-\hat{a}_{a b}-\hat{a}_{a} \hat{a}_{b}\right)
\end{aligned}
$$

Above, $\hat{R}_{a b}$ is the Ricci tensor constructed from the 3 -metric $\hat{h}_{a b}, \hat{R}=\hat{R}_{a}{ }^{a}$ is the scalar curvature, and $\hat{K}_{a b}$ denotes the extrinsic curvature, with $\hat{K}$ its trace. Also, $\hat{a}_{a}=\widehat{\nabla}_{a} \ln \hat{\alpha}$ is the acceleration of Eulerian observers, and we introduce the tensor $\hat{a}_{a b}=\widehat{\nabla}_{a} \hat{a}_{b}=\hat{a}_{b a}$, with $\hat{a}=\hat{a}_{a}{ }^{a}=\widehat{\nabla}_{a} \hat{a}^{a}$. Covariant differentiation with respect to $\hat{h}_{a b}$ is denoted by $\widehat{\nabla}_{a}$, and $\hat{\partial}_{o} \equiv \partial_{t}-£_{\hat{\beta}}$. The operator $\hat{\alpha}^{-1} \hat{\partial}_{o}$ is the derivative with respect to proper time along the normal to the space-like hypersurfaces, and $\hat{t}^{\mu}=\hat{\alpha} \hat{n}^{\mu}+\hat{\beta}^{\mu}$ is the time vector tangent to the congruence of world lines of coordinate observers. Equations (2) and (3) are respectively the Hamiltonian and momentum constraints. Equation (4), on the other hand, follows directly from the definition of the extrinsic curvature $\hat{K}_{a b}$. For simplicity, we are only considering vacuum space-times; however, it is straightforward to include matter sources.

The first step to derive the LNL formulation of Einstein equations involves splitting the evolution equations for the metric $\hat{h}_{a b}$ and extrinsic curvature $\hat{K}_{a b}$ into trace and trace-free equations. That is, using $\hat{K}_{a b}=\hat{A}_{a b}+\hat{h}_{a b} \hat{K} / 3$ with $\hat{A}_{a}{ }^{a}=0$, Eqs. (25) are rewritten as:

$$
\begin{aligned}
\hat{R}+\frac{2}{3} \hat{K}^{2}-\hat{A}_{a b} \hat{A}^{a b} & =0 \\
\hat{\nabla}_{b} \hat{A}^{a b}-\frac{2}{3} \hat{\nabla}^{a} \hat{K} & =0 \\
\hat{\partial}_{o} \ln \hat{h}^{1 / 2} & =-\hat{\alpha} \hat{K} \\
\hat{\partial}_{o} \hat{K}^{-\hat{\alpha}} \hat{K}^{2} & =\hat{\alpha}\left(\hat{R}-\hat{a}-\hat{a}_{a} \hat{a}^{a}\right) \\
\hat{h}^{1 / 3} \hat{\partial}_{o}\left(\hat{h}^{-1 / 3} \hat{h}_{a b}\right) & =-2 \hat{\alpha} \hat{A}_{a b} \\
\hat{\partial}_{o} \hat{A}_{a b}+2 \hat{\alpha} \hat{A}_{a c} \hat{A}^{c}{ }_{b}-\frac{1}{3} \hat{\alpha} \hat{A}_{a b} \hat{K} & =\hat{\alpha}\left\langle\hat{R}_{a b}-\hat{a}_{a b}-\hat{a}_{a} \hat{a}_{b}\right\rangle
\end{aligned}
$$

where angle brackets denote traceless symmetrization, i.e. $\left\langle T_{a b}\right\rangle \equiv T_{(a b)}-\frac{1}{3} h_{a b} T$ with $T=T_{a}{ }^{a}$.

In numerical relativity, conformal transformations have been extremely useful, in particular in the construction of initial data [8] to single out those "pieces" within $\hat{h}_{a b}$ and $\hat{A}_{a b}$ that are fixed by the constraints (6) and (7). Recently, conformal transformations have been considered also in formulations of the evolution equations (8-11). There are some indications that these formulations exhibit improvements in numerical stability properties [9]1]. Here, we introduce conformal transformation as a tool for factoring out nonlinearities. There is, however, a certain degree of ambiguity regarding the particular form of these transformation rules. Once the conformal transformation rule for the spatial metric $\hat{h}_{a b}$ is fixed, there is no specific recipe for choosing the remaining transformation rules for the extrinsic curvature $\hat{K}_{a b}$, lapse function $\hat{\alpha}$ and shift vector $\hat{\beta}^{a}$. For instance, in the construction of initial data, York's choices [8] were mostly aimed at simplifying the task of solving the constraints (6) and (耳). After several attempts, we found that the most convenient conformal transformations that yield a LNL factorization are:

$$
\begin{aligned}
\hat{h}_{a b} & =e^{4 \Omega} h_{a b} \\
\hat{h}^{1 / 2} & =e^{6 \Omega}
\end{aligned}
$$




$$
\begin{aligned}
\hat{A}_{a b} & =e^{2 \Omega} A_{a b} \\
\hat{K} & =e^{-2 \Omega} K \\
\hat{\alpha} & =e^{2 \Omega} \alpha \\
\hat{\beta}^{a} & =\beta^{a} .
\end{aligned}
$$

Conformal transformations similar to the above were used by Geroch [12] to study the structure of the gravitational field at infinity. The conformal transformation (12) for the spatial metric is just the standard rule used in most of the literature. The transformation (13) for the determinant of $\hat{h}_{a b}$ implies that $h=1$ [9-11]. This choice is always possible and simplifies the form of the equations while keeping the procedure general. A consequence of $h=1$, together with the conformal transformations (12-17), is that $h_{a b}$ is a tensor density of weight $-2 / 3$ and $A_{a b}$ a tensor density of weight $-1 / 3$. Similarly, $K$ is a scalar density of weight $1 / 3$ and $\alpha$ a scalar density of weight $-1 / 3$. Notice also that, contrary to the common practice of not assigning a conformal transformation to the trace of the extrinsic curvature $K$, we have chosen a conformal transformation for $K$ such that $\hat{K}_{a b}=e^{2 \Omega} K_{a b}$. It is also important to point out that with the above conformal transformations, the line element (11) takes the form

$$
d s^{2}=e^{4 \Omega}\left[-\alpha^{2} d t^{2}+h_{a b}\left(d x^{a}+\beta^{a} d t\right)\left(d x^{b}+\beta^{b} d t\right)\right] .
$$

Therefore, the space-time metric has the same transformation rule as the spatial metric. Furthermore, the transformations (12 17) yield quantities $\alpha$ and $\beta$ that are the lapse function and shift vectors of the conformal space-time, which would not necessarily be the case in general.

Given (12 17), the following conformal relationships are also obtained:

$$
\begin{aligned}
\hat{R}_{a b} & =R_{a b}-2 \omega_{a b}+4 \omega_{a} \omega_{b}-2 h_{a b}\left(\omega+2 \omega_{c} \omega^{c}\right) \\
\hat{R} & =e^{-4 \Omega}\left(R-8 \omega-8 \omega_{a} \omega^{a}\right) \\
\hat{a}_{a} & =a_{a}+2 \omega_{a} \\
\hat{a}_{a b} & =a_{a b}+2 \omega_{a b}-4 a_{(a} \omega_{b)}-8 \omega_{a} \omega_{b}+2 h_{a b}\left(a_{c} \omega^{c}+2 \omega_{c} \omega^{c}\right) \\
\hat{a} & =e^{-4 \Omega}\left(a+2 \omega+2 a_{a} \omega^{a}+4 \omega_{a} \omega^{a}\right),
\end{aligned}
$$

where $\omega_{a}=\nabla_{a} \Omega$ and $\omega_{a b}=\nabla_{a} \omega_{b}=\omega_{b a}$ with $\omega=\omega_{a}{ }^{a}=\nabla_{a} \omega^{a}$. We also have that $\hat{\partial}_{o}=\partial_{o}$ and $\left\langle\hat{\nabla}^{a} \hat{\beta}^{b}\right\rangle=$ $e^{-4 \Omega}\left\langle\nabla^{a} \beta^{b}\right\rangle$. With the set of conformal transformations (1223), Einstein equations (6-11) can now be rewritten as:

$$
\begin{aligned}
R+\frac{2}{3} K^{2}-A_{a b} A^{a b} & =8\left(\omega+\omega_{a} \omega^{a}\right) \\
\nabla_{b} A^{a b}-\frac{2}{3} \nabla^{a} K & =-4 \omega_{b}\left(A^{a b}+\frac{1}{3} h^{a b} K\right) \\
\partial_{o} \Omega & =-\frac{1}{6} \alpha K \\
\partial_{o} K-\frac{2}{3} \alpha K^{2} & =\alpha\left(R-a-a_{a} a^{a}-\Pi\right) \\
\partial_{o} h_{a b} & =-2 \alpha A_{a b} \\
\partial_{o} A_{a b}+2 \alpha A_{a c} A_{b}^{c}{ }_{b}-\frac{2}{3} \alpha A_{a b} K & =\alpha\left\langle R_{a b}-a_{a b}-a_{a} a_{b}-\Pi_{a b}\right\rangle,
\end{aligned}
$$

where

$$
\begin{aligned}
\Pi_{a b} & =4 \omega_{a b}-8 \omega_{a} \omega_{b}+2 h_{a b}\left(\omega+4 \omega_{c} \omega^{c}+a_{c} \omega^{c}\right) \\
\Pi & =h^{a b} \Pi_{a b}=10 \omega+16 \omega_{a} \omega^{a}+6 a_{a} \omega^{a} .
\end{aligned}
$$

In the form given by Eqs. (26 29), the Einstein evolutions equations are split into two sectors: $(\Omega, K)$ and $\left(h_{a b}, A_{a b}\right)$. The $(\Omega, K)$ sector is kept fully nonlinear, and, as we shall see later, approximations are introduced only when handling $\left(h_{a b}, A_{a b}\right)$, specifically in the transverse components of those fields. We start by rewriting the evolution equation (28) of the conformal metric as

$$
\partial_{t} h_{a b}-£_{\beta} h_{a b}=\partial_{t} h_{a b}-2\left\langle\nabla_{a} \beta_{b}\right\rangle=-2 \alpha A_{a b},
$$

where the substitution $£_{\beta} h_{a b}=2\left\langle\nabla_{a} \beta_{b}\right\rangle$ in the second equality uses the condition that $h_{a b}$ is a tensor density of weight $-2 / 3$. Next, we decompose the tensor $\alpha A_{a b}$ into its transverse and longitudinal parts, namely 


$$
\alpha A_{a b}=T_{a b}+L_{a b}
$$

such that $\nabla_{b} T^{a b}=T=0$ and $L_{a b}=\left\langle\nabla_{a} \xi_{b}\right\rangle$. Substitution of (33) into (32) yields

$$
\partial_{t} h_{a b}=2\left\langle\nabla_{a}\left(\beta_{b}-\xi_{b}\right)\right\rangle-2 T_{a b} .
$$

Since our goal is to treat the transverse-traceless fields perturbatively, it is then natural to choose the shift vector $\beta^{a}$ such that it eliminates the longitudinal terms in (32); that is, $\beta^{a}=\xi^{a}$. With this choice, Eq. (34) becomes

$$
\begin{aligned}
\partial_{t} h_{a b} & =-2 T_{a b} \\
L_{a b} & =\left\langle\nabla_{a} \beta_{b}\right\rangle .
\end{aligned}
$$

Notice that this choice of shift vector implies that the conformal space satisfies a gauge condition belonging to the class of "radiation gauges" [13], namely

$$
\nabla_{b} \partial_{t}\left(h^{N} h^{a b}\right)=0
$$

with $h=1$ and $N=1 / 3$. Condition (37), however, does not translate to a radiation gauge condition in the physical space-time since

$$
\widehat{\nabla}_{b} \partial_{t}\left(\hat{h}^{1 / 3} \hat{h}^{a b}\right)=\nabla_{b} \partial_{t} h^{a b}+10 \omega_{b} \partial_{t} h^{a b},
$$

and in general the second term of this equation does not vanish.

From the decomposition (33) and the transverseness of $T_{a b}$, it follows that

$$
\nabla_{b} L^{a b}=\nabla_{b}\left(\alpha A^{a b}\right)
$$

which can be rewritten using Eq. (25) as

$$
\nabla_{b} L^{a b}-\left(a_{b}-4 \omega_{b}\right)\left(L^{a b}+T^{a b}\right)=\frac{2}{3} \alpha\left(\nabla^{a} K-2 \omega^{a} K\right) .
$$

This equation is simply the momentum constraint and must be satisfied independent of the choice of shift vector. However, when the specific gauge choice (36) is made, Eq. (40) is alse viewed as a gauge condition from which the shift vector is constructed. A more geometrical interpretation of the gauge condition (40) is obtained by noticing that this condition, also known as "minimal strian gauge," can be derived from a global minimization of the time rate of change of the metric in the conformal space [13], that is, from the variational principle

$$
\delta S\left[\alpha, \beta^{a}\right]=\delta \int \partial_{t} h_{a b} \partial_{t} h_{c d} h^{a c} h^{b d} d v=0
$$

varying $\beta^{a}$. We must emphasize that condition (39), or equivalently (40), is a minimal strain gauge not applied to the physical space-time but to the conformal foliation.

The freedom of imposing a gauge condition in connection with the lapse function $\alpha$ still remains. For instance, if the choice is to have maximal conformal slices, i.e. $K=0$, Eq. (27) becomes an elliptic equation for $\alpha$, namely $a+a_{a} a^{a}=R-\Pi$. This condition also implies from Eq. (26) that $\partial_{o} \Omega=0$; that is, the conformal factor $\Omega$ is constant along the normals to the hypersurfaces. Other possible choices are the use the generalized harmonic gauge, $a_{o}+\alpha K=\alpha f$, with $f$ an arbitrary function, or a lapse function constructed from the variational principle (41) by varying $\alpha$ instead of $\beta^{a}$. The latter approach yields

$$
\alpha A_{a b} A^{a b}=\left\langle\nabla_{a} \beta_{b}\right\rangle A^{a b}
$$

which with the help of the Hamiltonian constraint (24) can be rewritten as:

$$
\alpha=\frac{L_{a b}\left(T^{a b}+L^{a b}\right)}{R+\frac{2}{3} K^{2}-8\left(\omega+\omega_{a} \omega^{a}\right)} .
$$

Notice that with this choice of gauge, the equations for the lapse and shift become a coupled set of equations [3].

So far, no approximations have been made. The theory remains completely nonlinear. The only condition is that the shift vector satisfies Eq. (40), a condition introduced to eliminate the longitudinal terms from the evolution equation of the conformal metric. We now assume that, in the physical systems where our LNL form of Einstein equations apply, 
the non-linearities are dominated by conformal (volume) deformations. As mentioned before, numerical simulations of neutron star binaries [4, 5, 14, 15] have shown that there is indeed a phase during the inspiral where this approximation holds. The main difference in the present work from those studies is that we allow for the presence of perturbations entering via shear deformations of a conformal background. That is, we decompose the conformal metric as

$$
h_{a b}=\gamma_{a b}+\Psi_{a b}
$$

with $\partial_{t} \gamma_{a b}=0$ and $\Psi_{a b}$ a perturbation. An immediate consequence of the assumption (44) is that from Eq. (35)

$$
\partial_{t} \Psi_{a b}=-2 T_{a b}
$$

so $T_{a b}$ becomes a perturbative quantity, in agreement with our goal of treating the "radiative," transverse-traceless fields as perturbations. Furthermore, having $\partial_{t} \gamma_{a b}=0$ and $T_{a b}$ transverse-traceless implies that Eq. (45) yields

$$
\begin{aligned}
\partial_{t} \Psi & =0 \\
\partial_{t} \widetilde{\nabla}_{b} \Psi^{a b} & =0,
\end{aligned}
$$

where $\Psi \equiv \gamma^{a b} \Psi_{a b}$. Above and from now on, tensors have their indices lowered and raised with the background metric $\gamma_{a b}$, and tildes denote quantities in connection with this metric. A direct implication of Eqs. (46) and (47) is that, if we choose initially the perturbation of the metric to be transverse and traceless, these conditions are preserved by the evolution equation (45). We will take advantage of this property and assume that initially $\widetilde{\nabla}_{b} \Psi^{a b}=\Psi=0$ since this choice simplifies the equations significantly.

At this point, we have the Hamiltonian constraint (24), the gauge condition (40) on the shift vector and the nonlinear evolution equations (26) and (27) for the conformal factor and the trace of the extrinsic curvature. In addition, we have the evolution equation (45) for the perturbation to the conformal metric; therefore, what remains is to obtain an evolution equation for $T_{a b}$.

From Eq. (45), we have that

$$
\partial_{o} \Psi_{a b}=-2\left(T_{a b}+\frac{1}{2} £_{\beta} \Psi_{a b}\right)=-2 \mathcal{T}_{a b}
$$

where we have defined

$$
\mathcal{T}_{a b} \equiv T_{a b}+\frac{1}{2} £_{\beta} \Psi_{a b} .
$$

On the other hand, Eq. 33 can be rewritten as:

$$
\begin{aligned}
\alpha A_{a b} & =L_{a b}+T_{a b}=\left\langle\nabla_{a} \beta_{b}\right\rangle+T_{a b} \\
& =\frac{1}{2} £_{\beta} h_{a b}+T_{a b}=\frac{1}{2} £_{\beta} \gamma_{a b}+\frac{1}{2} £_{\beta} \Psi_{a b}+T_{a b} \\
& =\left\langle\widetilde{\nabla}_{a} \beta_{b}\right\rangle+\mathcal{T}_{a b}=\tilde{L}_{a b}+\mathcal{T}_{a b},
\end{aligned}
$$

where we have used that $\gamma_{a b}$ is a tensor density of weight $-2 / 3$. Substitution of (50) into (29) then yields

$$
\begin{aligned}
& \partial_{o}\left(\alpha A_{a b}\right)-\left(a_{o}-4 \partial_{o} \Omega\right)\left(\alpha A_{a b}\right)+2\left(\alpha A_{a c}\right)\left(\alpha A_{b}^{c}\right)= \\
& \partial_{o} \tilde{L}_{a b}-\left(a_{o}-4 \partial_{o} \Omega\right) \tilde{L}_{a b}+2 \tilde{L}_{a c} \tilde{L}^{c}{ }_{b}+ \\
& \partial_{o} \mathcal{T}_{a b}-\left(a_{o}-4 \partial_{o} \Omega\right) \mathcal{T}_{a b}+2\left(\tilde{L}_{a c} \mathcal{T}^{c}{ }_{b}+\tilde{L}_{b c} \mathcal{T}^{c}{ }_{a}\right)-2 \tilde{L}_{a c} \tilde{L}_{d b} \Psi^{c d}= \\
& \alpha^{2}\left\langle R_{a b}-a_{a b}-a_{a} a_{b}-\Pi_{a b}\right\rangle,
\end{aligned}
$$

where

$$
\begin{aligned}
\left\langle a_{a b}\right\rangle & =\left\langle\tilde{a}_{a b}\right\rangle+\frac{1}{3}\left(\gamma_{a b} \Psi^{c d}-\gamma^{c d} \Psi_{a b}\right) \tilde{a}_{c d}-S_{a b}^{c} \tilde{a}_{c} \\
\left\langle a_{a} a_{b}\right\rangle & =\left\langle\tilde{a}_{a} \tilde{a}_{b}\right\rangle+\frac{1}{3}\left(\gamma_{a b} \Psi^{c d}-\gamma^{c d} \Psi_{a b}\right) \tilde{a}_{c} \tilde{a}_{d} \\
\left\langle R_{a b}\right\rangle & =\left\langle\tilde{R}_{a b}\right\rangle+\frac{1}{3}\left(\gamma_{a b} \Psi^{c d}-\gamma^{c d} \Psi_{a b}\right) \tilde{R}_{c d}-\frac{1}{2} \widetilde{\nabla}_{c} \widetilde{\nabla}^{c} \Psi_{a b}+\tilde{R}_{c(a} \Psi_{b)}{ }^{c}+\tilde{R}_{c a b d} \Psi^{c d} \\
\left\langle\Pi_{a b}\right\rangle & =\left\langle\tilde{\Pi}_{a b}\right\rangle+\frac{1}{3}\left(\gamma_{a b} \Psi^{c d}-\gamma^{c d} \Psi_{a b}\right) \tilde{\Pi}_{c d}-4 S^{c}{ }_{a b} \tilde{\omega}_{c}+2 \Psi_{a b}\left(\tilde{\omega}+4 \tilde{\omega}_{c} \tilde{\omega}^{c}+\tilde{a}_{c} \tilde{\omega}^{c}\right),
\end{aligned}
$$


with

$$
S_{b c}^{a}=\frac{1}{2}\left(\widetilde{\nabla}_{b} \Psi_{c}^{a}+\widetilde{\nabla}_{c} \Psi_{b}^{a}-\widetilde{\nabla}^{a} \Psi_{b c}\right)
$$

therefore, to zero-order Eq. (51) reads

$$
\partial_{o} \tilde{L}_{a b}-\left(a_{o}-4 \partial_{o} \Omega\right) \tilde{L}_{a b}+2 \tilde{L}_{a c} \tilde{L}_{b}^{c}=\alpha^{2}\left\langle\tilde{R}_{a b}-\tilde{a}_{a b}-\tilde{a}_{a} \tilde{a}_{b}-\tilde{\Pi}_{a b}\right\rangle .
$$

These evolution equations are redundant because, as mentioned before, the gauge conditions (40) that determine $\tilde{L}_{a b}$ are also the momentum contraints, which in turn preserve Eqs. (57). To first-order in the perturbations Eq. (51) yields the evolution equation for the perturbation $\mathcal{T}_{a b}$ :

$$
\begin{aligned}
& \partial_{o} \mathcal{T}_{a b}-\left(a_{o}-4 \partial_{o} \Omega\right) \mathcal{T}_{a b}+2\left(\tilde{L}_{a c} \mathcal{T}^{c}{ }_{b}+\tilde{L}_{b c} \mathcal{T}^{c}{ }_{a}\right)-2 \tilde{L}_{a c} \tilde{L}_{d b} \Psi^{c d}= \\
& \alpha^{2}\left[-\frac{1}{2} \widetilde{\nabla}_{c} \widetilde{\nabla}^{c} \Psi_{a b}+\tilde{R}_{c(a} \Psi_{b)}{ }^{c}+\tilde{R}_{c a b d} \Psi^{c d}+S^{c}{ }_{a b}\left(\tilde{a}_{c}+4 \tilde{\omega}_{c}\right)-2 \Psi_{a b}\left(\tilde{\omega}+4 \tilde{\omega}_{c} \tilde{\omega}^{c}+\tilde{a}_{c} \tilde{\omega}^{c}\right)\right. \\
& \left.+\frac{1}{3}\left(\gamma_{a b} \Psi^{c d}-\gamma^{c d} \Psi_{a b}\right)\left(\tilde{R}_{c d}-\tilde{a}_{c d}-\tilde{a}_{c} \tilde{a}_{d}-\tilde{\Pi}_{c d}\right)\right] .
\end{aligned}
$$

By combining this equation with Eq. (48), one obtains

$$
\left(-\frac{1}{\alpha} \partial_{o} \frac{1}{\alpha} \partial_{o}+\widetilde{\nabla}_{c} \widetilde{\nabla}^{c}\right) \Psi_{a b}=J_{a b}
$$

where

$$
\begin{aligned}
\frac{1}{2} \alpha^{2} J_{a b} & =\frac{2}{3} \alpha K \mathcal{T}_{a b}-2\left(\tilde{L}_{a c} \mathcal{T}^{c}{ }_{b}+\tilde{L}_{b c} \mathcal{T}^{c}{ }_{a}\right)+2 \tilde{L}_{a c} \tilde{L}_{d b} \Psi^{c d} \\
& +\alpha^{2}\left[\tilde{R}_{c(a} \Psi_{b)}{ }^{c}+\tilde{R}_{c a b d} \Psi^{c d}+S^{c}{ }_{a b}\left(\tilde{a}_{c}+4 \tilde{\omega}_{c}\right)-2 \Psi_{a b}\left(\tilde{\omega}+4 \tilde{\omega}_{c} \tilde{\omega}^{c}+\tilde{a}_{c} \tilde{\omega}^{c}\right)\right. \\
& \left.+\frac{1}{3}\left(\gamma_{a b} \Psi^{c d}-\gamma^{c d} \Psi_{a b}\right)\left(\tilde{R}_{c d}-\tilde{a}_{c d}-\tilde{a}_{c} \tilde{a}_{d}-\tilde{\Pi}_{c d}\right)\right]
\end{aligned}
$$

That is, the perturbation $\Psi_{a b}$ obeys a wave equation in the conformal space-time.

One more simplification of the LNL form of Einstein's equations is possible: neglecting the back-reaction of the perturbative fields $\left(\Psi_{a b}, \mathcal{T}_{a b}\right)$ on the nonlinear $(\Omega, K)$ and gauge $\left(\alpha, \beta^{a}\right)$ fields. Under this approximation, covariant differentiation and the raising and lowering of indices are performed with the background metric $\gamma_{a b}$.

In summary, the following picture emerges. Starting from the standard $3+1$ formulation of Einstein equations in terms of variables $\left(\hat{h}_{a b}, \hat{K}_{a b}\right)$, we have constructed a formulation in which the field variables are divided into:

1. Nonlinear fields $(\Omega, K)$ with their dynamics given by

$$
\begin{aligned}
\partial_{o} \Omega & =-\frac{1}{6} \alpha K \\
\partial_{o} K-\frac{2}{3} \alpha K^{2} & =\alpha\left(\tilde{R}-\tilde{a}-\tilde{a}_{a} \tilde{a}^{a}-\tilde{\Pi}\right) .
\end{aligned}
$$

2. Perturbative fields $\left(\Psi_{a b}, \mathcal{T}_{a b}\right)$ with equations of motion

$$
\begin{aligned}
\partial_{o} \Psi_{a b} & =-2 \mathcal{T}_{a b} \\
\partial_{o} \mathcal{T}_{a b}-a_{o} \mathcal{T}_{a b} & =-\frac{1}{2} \alpha^{2}\left(\widetilde{\nabla}_{c} \widetilde{\nabla}^{c} \Psi_{a b}-J_{a b}\right) .
\end{aligned}
$$

3. A background conformal metric $\gamma_{a b}$ such that $\partial_{t} \gamma_{a b}=0$. 
4. And a shift vector $\beta^{a}$ satisfying the condition

$$
\widetilde{\nabla}_{b} \tilde{L}^{a b}-\left(\tilde{a}_{b}-4 \tilde{\omega}_{b}\right) \tilde{L}^{a b}=\frac{2}{3} \alpha\left(\widetilde{\nabla}^{a} K-2 \tilde{\omega}^{a} K\right) .
$$

The conditions and assumptions used to derive this LNL system of equations are:

1. The field variables are conformally transformed according to (12 17$)$.

2. The shift vector is chosen to provide the longitudinal part of $\alpha A_{a b}$, which in turns implies that it must satisfy Eq. (65).

3. The conformal metric $h_{a b}$ is decomposed into a background metric $\gamma_{a b}$ plus a transverse-traceless perturbation $\Psi_{a b}$.

4. The back-reaction from the perturbations is ignored.

As mentioned before, the only gauge freedom remaining is that in connection with the lapse function $\alpha$. Furthermore, if a fully constrained evolution is preferred, the conformal factor could be obtained from the Hamiltonian constraint (24). When in addition one assumes that the metric perturbation $\Psi_{a b}$, and consequently $\mathcal{T}_{a b}$, could be neglected, the LNL system reduces to a nonlinear system similar to that used by Wilson and collaborators [4. star collisions. The differences would be due to our particular choice of conformal transformations.

In conclusion, with the help of suitable conformal transformations and a shift vector gauge condition, we have constructed an approximate formulation of Einstein equations that could yield advantages in the study of gravitational systems where radiative, transverse-traceless variables can be treated as perturbations of a fixed, background spatial metric, with the remaining fields possessing nonlinear dynamics. Since this separation depends on the choice of conformal transformations, it remains to be tested whether the conformal transformations used in our derivation of the LNL system of equations indeed constitute an appropriate approximation. When the LNL system is applied to the Inspiral-Merging intermediate regime, an interesting approach suggested in Ref. [3] that could place our approximation in a more firmer grounds, is to perform the calculations in a co-rotating frame. The objective under this approach is to find a spatial vector $b^{\mu}$, i.e. $b_{\mu} n^{\mu}=0$ with $n^{\mu}$ the normal to the constant $t$ hypersurfaces, such that $\xi^{\mu}=\alpha n^{\mu}+\beta^{\mu}+b^{\mu}$ is an "almost Killing vector field," namely $£_{\xi} g_{\mu \nu} \approx 0$.

Special thanks to Abhay Ashtekar, Mijan Huq, Richard Matzner, Philip Papadopoulos and Deirdre Shoemaker for helpful discussions. This work was supported by NSF grants PHY 98-00973 and PHY 93-57219 (NYI).

[1] R.H. Price and J. Pullin Phys. Rev. Lett. , 72, 3297 (1994)

[2] For current status of the Binary Black Hole Grand Challenge, see http://www.npac.syr.edy/projects/bh

[3] P.R. Brady, J.D.E. Creighton and K.S. Thorne Phys. Rev. D, 58, 061501 (1998)

[4] J.R. Wilson and G.J. Mathews, Phys. Rev. Lett. , 75, 4161 (1995)

[5] J.R. Wilson, G.J. Mathews and P. Marronetti, Phys. Rev. D, 54, 1317 (1996)

[6] E.E. Flanagan, Phys. Rev. Lett. , 82, 1354 (1999)

[7] R. Arnowitt, S. Deser and C.W. Misner: in Gravitation ed. L. Witten (Wiley, New York, 1962) p. 227.

[8] J.W. York, Jr., in sl Sources of Gravitational Radiation, ed. L. Smarr (Cambridge University Press, Cambridge, England, 1979), p. 83.

[9] M. Shibata and T. Nakamura, Phys. Rev. D, 52, 5428 (1995)

[10] T.W. Baumgarte and S.L. Shapiro, Phys. Rev. D, 59, 024007 (1999)

[11] M. Alcubierre, B. Brügmann, M. Miller and W.-M. Suen gr-qc/9903030

[12] R. Geroch J. Math. Phys., 13, 956 (1972)

[13] L. Smarr and J.W. York, Jr., Phys. Rev. D, 17, 1945 (1978)

[14] T.W. Baumgarte, G.B. Cook, M.A. Scheel, S.L. Shapiro and S.A. Teukolsky, Phys. Rev. Lett. , 79, 1182 (1997)

[15] S. Bonazzola, E. Gourgoulhon and J.-A. Marck, Phys. Rev. Lett. , 82, 892 (1999) 